\section{Posterior Reversible Encephalopathy Syndrome after Paralytic Ileus during B-Cell Precursor Acute Lymphoblastic Leukemia Induction Therapy}

Keywords: Acute lymphoblastic leukemia; Paralytic ileus; Posterior reversible encephalopathy syndrome; Vincristine

\begin{abstract}
A 9 year-old boy with B-cell precursor acute lymphoblastic leukemia developed paralytic ileus and severe abdominal pain during the induction therapy. In spite of fentanyl administration, abdominal pain could not be controlled sufficiently. On $31^{\text {st }}$ day, he had convulsion and second convulsion with sudden severe visual impairment on 33rd day, although his blood pressure was controlled within normal limit. Magnetic resonance imaging revealed hyperintensity lesions in the bilateral frontal and occipital lobes on T2-weighted images and he was diagnosed as having posterior reversible encephalopathy syndrome. His visual activity returned along with an improvement of paralytic ileus and colic pain. In addition to the control of blood pressure, the management of paralytic ileus and pain control are important for the prevention and treatment of posterior reversible encephalopathy syndrome.
\end{abstract}

\section{Introduction}

Posterior Reversible Encephalopathy Syndrome (PRES) has been reported as central nervous system complication during chemotherapy [1]. It is characterized by typical neurological symptoms such as headache, mental disturbance, seizure and visual impairment, and characteristic Magnetic Resonance Imaging (MRI) findings predominantly found in the posterior lobes [2]. Symptoms and radiological findings usually recover in more than $90 \%$ of the cases, but permanent neurological symptoms remain in the other cases [1]. Although the mechanism is not clearly understood, hypertension and chemotherapeutic agents are suspected risk factors.

\section{Case Report}

A 9 year-old boy diagnosed as having B-Cell Precursor Acute Lymphoblastic Leukemia (BCP-ALL) with ETV6-AML1 fusion gene received induction chemotherapy according to Japan Pediatric Leukemia Study Group (JPLSG) B12 Standard Risk Protocol with permission from the guardians. He presented paralytic ileus and abdominal pain on day 19 and required pain control therapy with fentanyl administration (Figure 1). On day 21, hyponatremia and Clostridium difficile associated diarrhea appeared and cefepime was started for febrile neutropenia on day 23. Vincristine-induced SIADH was suspected and prophylactic itraconazole was stopped to avoid the interaction with vincristine. On day 27 , his abdominal pain worsened and then massive hematuria was noticed on day 30 . He had convulsion

\section{Journal of}

Hematology \& Thrombosis

\author{
Noriko Mitsuiki ${ }^{1}$, Chika Kobayashi ${ }^{1}$, Keita \\ Tamanuki $^{2}$, Kenj Kobayashi ${ }^{2}$, Yoshiho Hatai ${ }^{2}$, Jun \\ Kohyama $^{2}$ and Masayuki Nagasawa ${ }^{1,3 *}$
}

${ }^{\prime}$ Tokyo Bay Urayasu-Ichikawa Medical Center, Division of Hematology, Oncology and Immunology, Japan

${ }^{2}$ Tokyo Bay Urayasu-Ichikawa Medical Center, Department of Pediatrics, Japan

${ }^{3}$ Musasino Red Cross Hospital, Department of Pediatrics, Japan

\section{*Address for Correspondence:}

Masayuki Nagasawa, Department of Pediatrics, Musashino Red Cross Hospital 1-26-1, Kyonan-cho, Musashino-city, Tokyo 1808610, Japan, Fax: +81-0422-32-3525; Tel: +81-0422-32-3111; E-mail: mnagasawa.ped@tmd.ac.jp

Submission: 01 November, 2017 Accepted: 30 November, 2017 Published: 04 December, 2017

Copyright: () 2017 Mitsuiki N, et al. This is an open access article distributed under the Creative Commons Attribution License, which permits unrestricted use, distribution, and reproduction in any medium, provided the original work is properly cited.

on day 31. Magnetic Resonance Imaging (MRI) T2-Weighted Images (T2WI) revealed bilateral subcortical and cortical hyperintensity lesions in the occipital lobes (Figure 2a) and MR angiography showed partial vascular stenosis. Abdominal CT scan revealed bilateral urolithiasis in the pelvis with no hydronephrosis. His blood pressure was within normal limits and heparin and aspirin were started in consideration of PRES or cerebral infarction. Fourth vincristine, $8^{\text {th }}$ asparaginase, and second intrathecal tripple injection were cancelled. Intermittent colic stomach and abdominal pain persisted and then he had generalized tonic seizure again followed by sudden severe visual

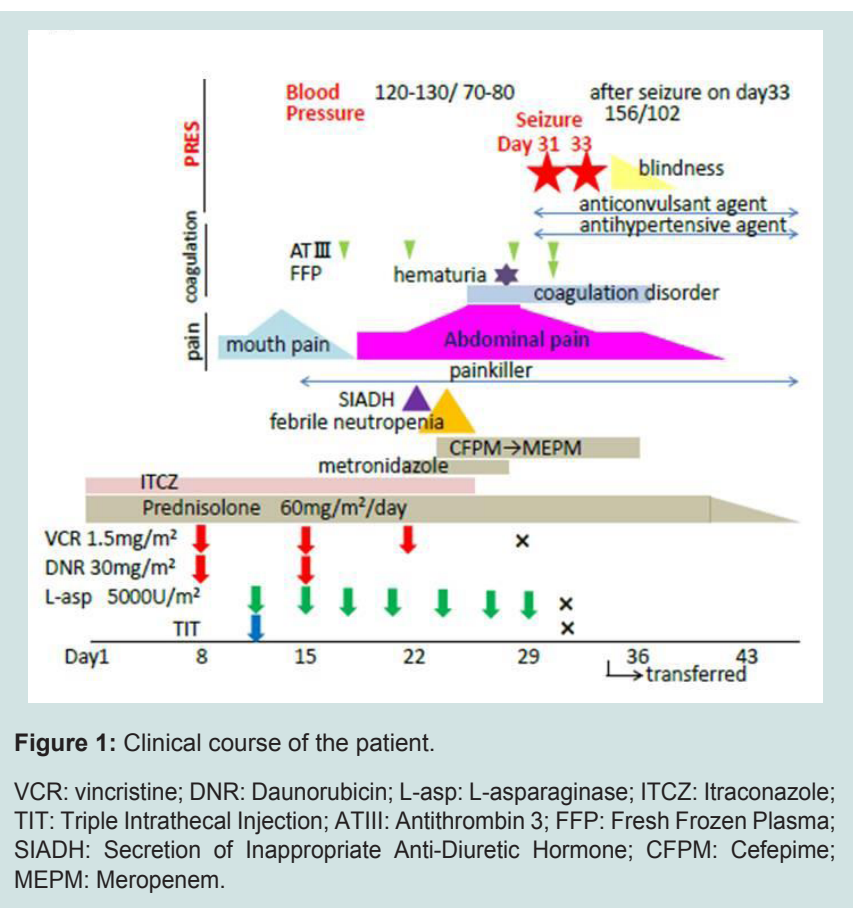


Citation: Mitsuiki N, Kobayashi C, Tamanuki K, Kobayashi K, Hatai Y, et al. Posterior Reversible Encephalopathy Syndrome after Paralytic Ileus during B-Cell Precursor Acute Lymphoblastic Leukemia Induction Therapy. J Hematol Thromb 2017;3(1): 3.

impairment on day 33. The blood examination revealed that white blood cells of $7700 / \mu \mathrm{L}$, hemoglobin of $8.9 \mathrm{~g} / \mathrm{dL}$, platelet of $7.8 \times 104 /$ $\mu \mathrm{L}$, urea nitrogen of $15.1 \mathrm{mg} / \mathrm{dL}$, creatinine of $0.35 \mathrm{mg} / \mathrm{dL}$, LDH of $564 \mathrm{U} / \mathrm{L}, \mathrm{AST}$ of $40 \mathrm{U} / \mathrm{L}, \mathrm{ALT}$ of $26 \mathrm{U} / \mathrm{L}, \mathrm{Na}$ of $127 \mathrm{mEq} / \mathrm{L}, \mathrm{CRP}$ of $0.69 \mathrm{mg} / \mathrm{dL}$, antithrombin 3 of $75 \%$, Protein C of $63 \%$, Protein $\mathrm{S}$ of $61.1 \%$, PT-INR of 1.27 , and d-dimer of $5.9 \mu \mathrm{g} / \mathrm{mL}$. His blood pressure was increased after second seizure up to $156 / 102 \mathrm{mmHg}$. MRI T2WI revealed new hyperintensity lesions in the bilateral frontal and occipital lobes (Figure 2b). Cerebrospinal fluid revealed no abnormal findings except for a slight increased protein of 57.9 $\mathrm{mg} / \mathrm{dL}$. He received blood pressure control therapy in the intensive

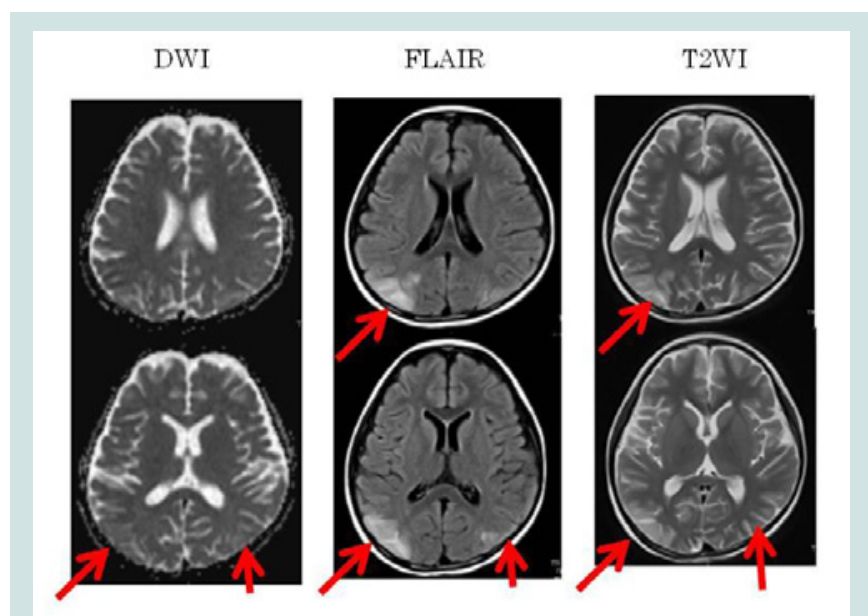

Figure 2a: MRI images after the first Convulsion and second convulsion.

ADC, FLAIR, and T2WI maps of MRI were presented. Bilateral subcortical and cortical hyperintensity lesions were noticed in the frontal and occipital lobes (red arrows)

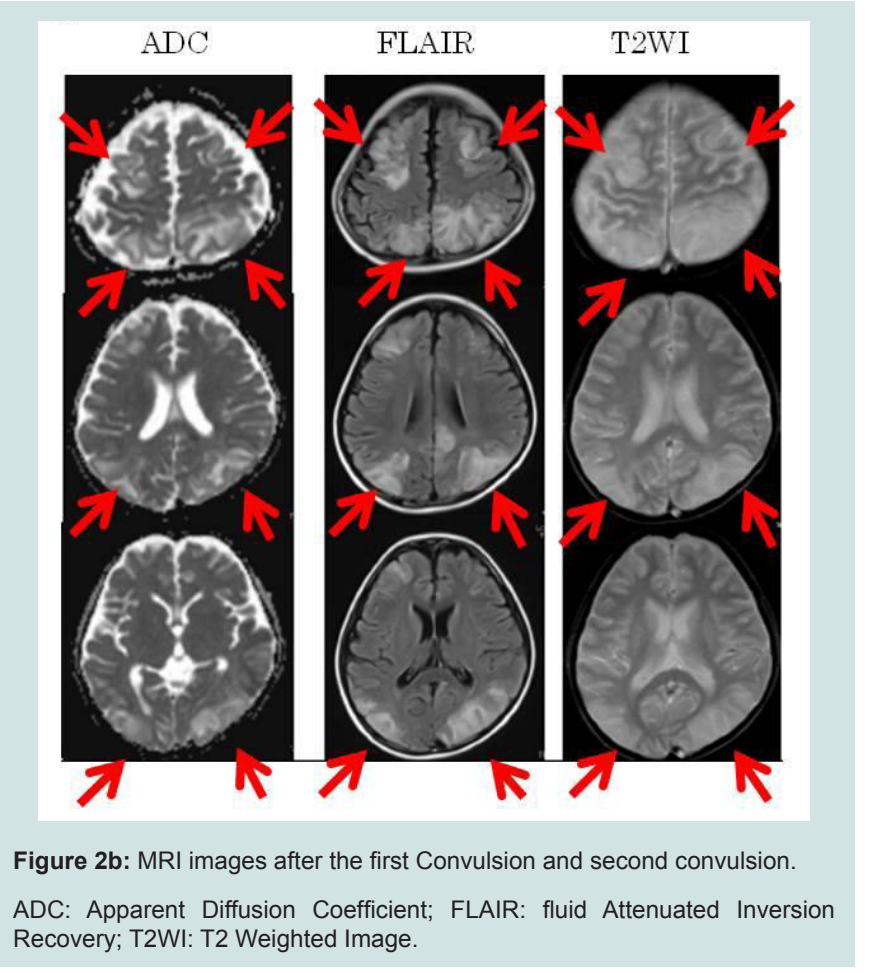

care unit. His visual activity returned along with an improvement of paralytic ileus and colic pain. Although he required levetiracetam as an anti-convulsant to control PRES-associated secondary epilepsy, he recovered without other significant neurological sequel and complete remission was confirmed two weeks later. He has received subsequent scheduled chemotherapy of BCP-ALL and kept complete remission. On behalf of the patient, informed consent was obtained from his parents for this case report.

\section{Discussion}

PRES is a clinical syndrome characterized by headache, confusion, various visual abnormalities, and seizure with finding of neurological imaging indicating the existence of edema in the posterior regions of the brain. Although, the pathogenesis of PRES is not clearly understood, PRES is caused by various heterogenous factors, the commonest being hypertension, followed by eclampsia, hematopoietic stem cell transplantation, organ transplantation, sepsis, autoimmune disease, immunosuppressive therapy and anti-cancer chemotherapy [3]. Furthermore, atypical PRES variants associated with neoplastic process itself, obstructive hydrocephalus or brain surgery have been reported [4-6]. Hypertension affects a cerebrovascular autoregulation function and raises capillary pressure and may present with vascular endothelial damages [7]. However, PRES will occur without hypertension if vascular endothelial damages are induced from other causes. In fact, blood pressure in the adults is higher than the children, but it is reported that PRES is more common in children. It might be due to the lack of autoregulation in children rather than in adults.

L-asparaginase; a key drug used for the treatment of BCP-ALL possibly induces micro-thrombi formation and direct endothelial damages [8]. While PRES is usually induced during the initial induction chemotherapy in the hematological malignancy, it occurs after multiple chemotherapies in the solid tumor [9]. In addition to the endothelial damages by anticancer drugs, the susceptibility of tumor cells to chemotherapy may account for this difference. In the hematological malignancy, quantity of tumor suddenly decreases after initial chemotherapy and the release of cellular components with accompanying cytokines may lead to further endothelial damages [10].

As a pathogenesis of paralytic ileus in this case, the interaction between vincristine and itraconazole must be taken into account. Both of drugs are metabolized with CYP3A4, and thereby side effects of vincristine might be reinforced by the elevation of blood concentration through the interaction [11]. Some cases of PRES after paralytic ileus induced by vincristine are reported [12,13]. It is inferred that abnormal brain blood vessel shrinkage by the sudden changes of abdominal pressure and an effect on the sympathetic innerved backward circulation due to the autonomic nervous system disorder are the mechanisms of pathogenesis [12]. In this setting, incidentally found urolithiasis in this patient which was not detected before the start of chemotherapy might be associated with his severe intractable colic pain and consequent PRES.

We suggest that the management of paralytic ileus and pain control are important for the prevention and treatment of PRES in addition to the control of blood pressure during the induction therapy for ALL. 
Citation: Mitsuiki N, Kobayashi C, Tamanuki K, Kobayashi K, Hatai Y, et al. Posterior Reversible Encephalopathy Syndrome after Paralytic Ileus during B-Cell Precursor Acute Lymphoblastic Leukemia Induction Therapy. J Hematol Thromb 2017;3(1): 3.

\section{References}

1. de Laat P, Te Winkel ML, Devos AS, Catsman-Berrevoets CE, Pieters $R$ et al. ( 2011) Posterior reversible encephalopathy syndrome in childhood cancer. Ann Oncol 22: 472-478.

2. Hinchey J, Chaves C, Appignani B, Breen J, Pao L, et al. (1996) A reversible posterior leukoencephalopathy syndrome. N Engl J Med 334: 494-500.

3. Bartynski WS (2008) Posterior reversible encephalopathy syndrome, part 1: fundamental imaging and clinical features. AJNR Am J Neuroradio 29: 10361042.

4. Aureli V, Giammattei L, Maduri R, Daniel RT, Messerer M (2017) Posterior reversible encephalopathy syndrome (PRES) due to neuroblastoma in a child presenting with acute hydrocephalus. Childs Nerv Syst.

5. Naval Baudin P, Martínez Fernández T, Mora P, Corrales Cruz A, Sardiñas Barrero JC, et al. (2017) Posterior reversible encephalopathy syndrome (PRES) with obstructive hydrocephalus. Diagn Interv Imaging 98: 745-746.

6. Gonzalez Quarante LH, Mena-Bernal JH, Martin BP, Ramirez Carrasco M, Munoz Casado MJ, et al. (2016) Posterior reversible encephalopathy syndrome (PRES): a rare condition after resection of posterior fossa tumors: two new cases and review of the literature. Childs Nerv Syst 32: 857-863.

7. Garg RK, Malhotra HS, Patil TB, Agrawal A (2013) Cerebral-autoregulatory dysfunction syndrome. BMJ Case Rep.
8. Schneider P, Van Dreden P, Rousseau A, Kassim Y, Legrand E, et al. (2010) Increased levels of tissue factor activity and procoagulant phospholipids during treatment of children with acute lymphoblastic leukaemia. $\mathrm{Br} \mathrm{J}$ Haematol 148: 582-592

9. Imai H, Okuno N, Ishihara S, Nakano S, Higuchi S, et al. (2012) Reversible posterior leukoencephalopathy syndrome after carboplatin and paclitaxel regimen for lung cancer. Intern Med 51: 911-915.

10. Ozkan A, Hakyemez B, Ozkalemkas F, Ali R, Ozkocaman V, et al. (2006) Tumor lysis syndrome as a contributory factor to the development of reversible posterior leukoencephalopathy. Neuroradiology 48: 887-892.

11. Teusink AC, Ragucci D, Shatat IF, Kalpatthi R (2012) Potentiation of vincristine toxicity with concomitant fluconazole prophylaxis in children with acute lymphoblastic leukemia. Pediatr Hematol Oncol 29: 62-67.

12. Panis B, Vlaar AM, van Well GT, Granzen B, Weber JW, et al. (2010) Posterior reversible encephalopathy syndrome in paediatric leukaemia. Eur J Paediatr Neurol 14: 539-545.

13. Tsukamoto S, Takeuchi M, Kawajiri C, Tanaka S, Nagao Y, et al. (2017) Posterior reversible encephalopathy syndrome in an adult patient with acute lymphoblastic leukemia after remission induction chemotherapy. Int J Hematol 95: 204-208. 